\title{
ESTUDOS SOBRE A NUTRIÇÃO MINERAL DO MILHO. II - DEFICIÊNCIAS DE MACRONUTRIENTES NA VARIEDADE PIRANÃO*
}

E. MALAVOLTA** V.M. RAPCHAN **

A.M.G. DE CASTRO***

A.P. SOUZA***

E.S. LOPES ***

L.F. SOBRAL ***

L.A. LOVATO***

M. MENEZES***

O. TISSELLI FỌ***

G.D. OLIVEIRA**

\section{RESUMO}

Foram induzidos sintomas de carência de macronutrientes no mitho, var. Piranão, exceto no caso do enxofre.

Os níveis foliares de $\mathrm{N}, \mathrm{P}, \mathrm{K}, \mathrm{Ca}, \mathrm{Mg}$ e $\mathrm{S}$ foram determinados nas plantas sujeitos à nutrição completa e às deficiências de macronutrientes.

\section{INTRODUÇÄO}

Estudos de nutrição mineral das plantas cultivạdas são freqüentemente necessários para dar bases para a adubação adequada das culturas.

Uma revisão da literatura não mostrou existirem trabalhos sistemáticos sobre os efeitos das carências de macronutrientes no milho.

AGBOOLA (1967), ARRUDA (1968) e SHAH (1969), verificaram que o suprimento de $\mathrm{N}, \mathrm{P}, \mathrm{K}$ ao milho aumentou significativamente a produção de grãos e também o peso da espiga. No entanto, PUNT AMKER et al. (1965), LAIRD \& RODRIGUEZ (1965), RATHORE \& SINGH (1967) tiveram melhores produções com adubação de $\mathrm{N}$ do que com outros elementos. Por sua vez, SAXENA \& GAUTAM (1971), MALIK \& SHAH (1968), ANDERSON (1970), concluíram que a combinação N e P alcançou resultados significativos na produção e também no conteúdo de proteína.

Ressaite-se a importância do $\mathrm{P}$ na vida da planta, pois segundo WALLACE \& DEUTSH (1968) sua deficiência acarreta distúrbios, tais como, baixa transpiração e diminuição da circulação de água, além de diminuir a capacidade de abertura dos estômatos.

\footnotetext{
* Entregue para publicação em 16/12/1975. Com ajuda da EMBRAPA, CNEN, CNPq, FAPESP e BNDE.

* Departamento de Química e CENA, ESALQ/USP, Piracicaba.

* * Estudantes Pós-graduados de Solos e Nutrição de Plantas, ESALQ/USP, Piracicaba.
} 
NAKANO \& JUSHIBIKI (1967) trabalhando com Mg, em milho, obtiveram aumentos no desenvolvimento da planta, nas espigas e na produção de grãos, quando adicionaram o citado elemento. Por sua vez, AGBIM (1968), mostrou que o P reduziu a concentração e absorção de $\mathrm{Zn}$, mas aumentou a concentração e absorção de $\mathrm{Ca}$ e $\mathrm{Mg}$.

REHM \& CALDWELL (1970), verificaram que a influência de qualquer fonte de $\mathrm{N}$ na absorção de $\mathrm{S}$ é o resultado de reações ou processos fisiológicos dentro da planta. Por outro lado, PUNT AMKER et al. (1965) elevando o suprimento de $\mathrm{N}$ a $120 \mathrm{~kg} / \mathrm{ha}$, obtiveram um aumento nas concentrações de $\mathrm{N}$ em todas as partes da planta e em todos os estágios de crescimento. Aplicando $\mathrm{N}$ no início do desenvolvimento, a absorção de $\mathrm{P}$ e $\mathrm{K}$ foi intensificada.

GALVÃO et al. (1969) concluíram que a população de plantas teve menos efeito sobre a produção do que a adubação nitrogenada e que o nitrogênio aumentou significativamente o peso médio das espigas.

BRAGA (1968) utilizando o milho como planta indicadora para avaliar a fertilidade do solo encontrou uma co:relação significativa entre peso verde e seco.

GALLO et al. (1968), encontraram correlações significativas entre o teor de macronutrientes nas folhas e a produção; no entanto, os autores ressalvam que alguns fatores influenciam na generalização dos resultados para nitrogênio e potássio.

MIRANDA \& MIRANDA (1971) através da realização de vários ensaios conseguiram ajustar uma função para as relações entre adubação e produção que permite fazer-se recomendações de adubação com base na análise dos solos.

SOBRAL (1972), estudou os efeitos de níveis crescentes de P em um PVA pobre em fósforo concluindo que em ausência de adubação fosfatada as plantas não cresceram e mostraram os sintomas característicos de deficiência do elemento.

A T.V.A. (1971) publicou uma revisão de 653 trabalhos relacionados com a nutrição do milho, cuja consulta é obrigatória.

ANDRADE (1975) estudou a marcha de absorção de nutrientes em cinco cultivares de milho em condições de campo.

$O$ presente trabalho teve como objetivo estudar os sintomas de deficiência de macronutrientes (N, P, K, Ca, Mg e S) em plantas de milho (Zea mays L.) cv. Piranão cultivadas em solução nutritiva, bem como verificar os referidos elementos nas folhas superiores e inferiores das mesmas, para se obter padrões do estado nutricional.

\section{MATERIAS E MÉTODOS}

O trabalho de deficiência foi conduzido em casa-de-vegetação, tendo sido empregadas plantas de milho (Zea mays L.) cv. Piranão; as sementes foram fornecidas peio Prof. Dr. E. Paterniani, Departamento de Genética, ESALQ/USP, Piracicaba, que obteve o cultivar. 
As plantas para o presente ensaio foram obtidas da seguinte maneira: fez-se o se. meio em bandejas contendo vermiculita, umedecida com água de torneira. Após a germinação, as plantas foram transferidas para bandejas contendo solução nutritiva completa de HOAGLAND \& ARNON (1950), diluída a 1:5. Decorrido um período de 14 dias da semeadura fez-se a transferência das piantas para vasos plásticos com capacidade para 2,5 litros, contendo solução completa com a seguinte composição:

\begin{tabular}{|c|c|}
\hline Soluçōes & $\mathrm{ml} /$ vaso \\
\hline $\mathrm{KH}_{2} \mathrm{PO}_{4} \mathrm{M}$ & 1 \\
\hline $\mathrm{KNO}_{3} \mathrm{M} \ldots$ & 5 \\
\hline $\mathrm{Ca}\left(\mathrm{NO}_{3}\right) 2.4 \mathrm{H}_{2} \mathrm{OM}$ & 5 \\
\hline $\mathrm{MgSO}_{4} .7 \mathrm{H}_{2} \mathrm{OM} \ldots$ & 2 \\
\hline Sol. "a" . . . . . & 1 \\
\hline EDTA-Fe ... & 1 \\
\hline
\end{tabular}

Vale ressaltar, que a solução "a" apresentava a seguinte composição:

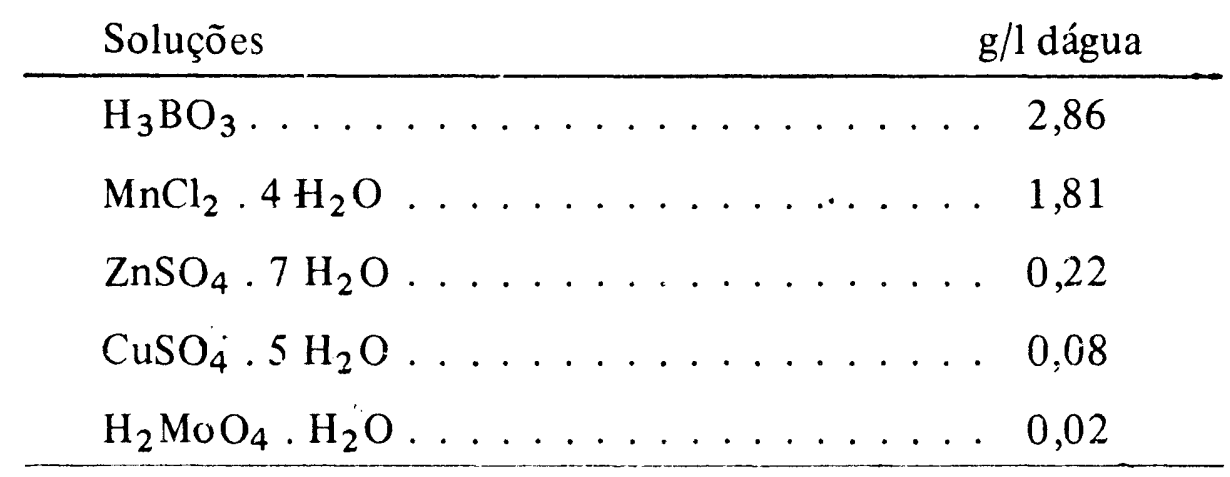

Após uma semana de cultivo das plantas nas soluções mencionadas procedeu-se o in ício do ensaio de deficiência de macronutrientes, sendo as soluções preparadas, conforme o Quadro 1 .

Decorrido um periodo de 15 dias de permanência das plantas em vasos de 2,5 litros de capacidade, as mesmas foram transferidas para vasos de 20 litros, exceto uma das repetições do tratamento $-\mathrm{N}$, que foi colocada em vaso com capacidade para 8 litros.

As soluções nutritivas foram substituídas a cada 15 dias e, durante a duração do ensaio manteve-se um sistema de arejamento constante.

O delineamento utilizado foi o inteiramente casualizado com 7 tratamentos e duas repetições.

Durante o período de condução do experimento foram efetuadas, semanalmente medições de altura das plantas e contagens do número dè folhas.

As plantas foram pulverizadas com Aldrin e Thiordan, visando o controle, respectivamente da lagarta do cartucho (Spodoptera frugiperda) e ácaros. 
QUADRO 1 - Composição das soluções nutritivas utilizadas no ensaio.

\begin{tabular}{|c|c|c|c|c|c|c|c|}
\hline \multirow{2}{*}{ Soluções } & \multicolumn{7}{|c|}{$\mathrm{mi} /$ /itro de soluçāo nutritiva } \\
\hline & $-\mathrm{N}$ & $-\mathrm{K}$ & $-\mathrm{P}$ & $-\mathrm{Mg}$ & $-\mathrm{Ca}$ & $-\mathrm{S}$ & completa \\
\hline $\mathrm{K}_{2} \mathrm{SO}_{4} 0,5 \mathrm{M}$ (p.m. 174,27) & 5 & 0 & 0 & 3 & 0 & 0 & 0 \\
\hline $\mathrm{MgSO}_{4} .7 \mathrm{H}_{2} \mathrm{OM}$ (p.m. 246,48) & 2 & 2 & 2 & 0 & 2 & 0 & 2 \\
\hline $\mathrm{Ca}\left(\mathrm{H}_{2} \mathrm{PO}_{4}\right)_{2} \cdot \mathrm{H}_{2} \mathrm{O} 0,05 \mathrm{M}$ & 10 & 10 & 0 & 0 & 0 & 0 & 0 \\
\hline $\mathrm{CaSO}_{4}$ 0,01 M (p.m. 136,14) & 200 & 0 & 0 & 0 & 0 & 0 & 0 \\
\hline $\mathrm{Ca}\left(\mathrm{NO}_{3}\right)_{2} .4 \mathrm{H}_{2} \mathrm{OM}$ & 0 & 5 & 4 & 4 & 0 & 4 & 5 \\
\hline $\mathrm{KNO}_{3} \mathrm{M}$ (p.m. 101,11) & 0 & 0 & 6 & 6 & 5 & 6 & 5 \\
\hline $\mathrm{KH}_{2} \mathrm{PO}_{4} \mathrm{M}$ (p.m. 136,09) & 0 & 0 & 0 & 1 & 1 & 1 & 1 \\
\hline $\mathrm{Mg}\left(\mathrm{NO}_{3}\right)_{2} .6 \mathrm{H}_{2} \mathrm{O} \mathrm{Mi}$ (p.m. 256,41) & 0 & 0 & 0 & 0 & 0 & 2 & 0 \\
\hline Micro & 1 & 1 & 1 & 1 & 1 & 1 & 1 \\
\hline
\end{tabular}

Após o aparecimento dos sintomas de deficiência nos tratamentos $-\mathrm{N},-\mathrm{P},-\mathrm{K},-\mathrm{Ca}$, - $\mathrm{Mg}$, as plantas foram colhidas, tendo-se o cuidado de separar: raiz, colmo, folhas inferiores e superiores e pendão. Com relação aos tratamentos -S e completo a colheita foi efetuacia em época posterior, em virtude de se objetivar a obtenção da produção.

$\mathrm{O}$ material colhido foi secado a $70^{\circ} \mathrm{C}$, com circulação de ar, durante uma semana, aproximadamente. Em seguida procedeu-se a pesagem e moagem do material no qual foram determinados $\mathrm{N}, \mathrm{P}, \mathrm{K}, \mathrm{Mg}$ e $\mathrm{S}$ tanto nas folhas superiores quanto nas inferiores, conforme os métodos seguintes:

$\mathrm{N}$ : método de Kjedahl

P: determinação colorimétrica em extrato nitroperclórico, cor desenvolvida pelo reagente misturado vanadato-molibdato

K, Ca, Mg: determinação no mesmo extrato pelo espectrofotômetro de absorção atômica

S: determinação gravimétrica baseada na precipitação do $\mathrm{S}$ pelo $\mathrm{BaCl}_{2}$ na forma de $\mathrm{BaSO}_{4}$.

Para fins de cálculos estatísticos a matéria seca produzida foi dividida em raízes $\mathrm{c}$ parte aérea, tendo sido efetuada análise de variância e a comparação das médias pelo teste de Tukey.

Do mesmo modo, foi efetuada a análise estatística para os resultados das análises químicas. 


\section{RESULTADOS E DISCUSSÃO}

\section{Altura das plantas e número de folhas}

Os resultados concernentes a medições de altura e contagens do número de folhas nos diferentes tratamentos são apresentados no Quadro 2.

QUADRO 2 - Altura $(\mathrm{cm})$ e número de folhas das plantas de milho (Zea mays L.) cv. Piranão cultivadas em solução nutritiva.

\begin{tabular}{|c|c|c|c|c|c|c|c|c|c|c|c|c|}
\hline \multirow[b]{3}{*}{ Tratamentos } & \multicolumn{12}{|c|}{ Semanas } \\
\hline & \multicolumn{2}{|c|}{$1 \mathrm{a}$} & \multicolumn{2}{|c|}{$2 \mathrm{a}$} & \multicolumn{2}{|c|}{$3 a$} & \multicolumn{2}{|c|}{$4 a$} & \multicolumn{2}{|c|}{$5 a$} & \multicolumn{2}{|c|}{$6 ?$} \\
\hline & A & $\mathrm{NF}$ & A & $\mathrm{NF}$ & A & $\mathrm{NF}$ & A & $\mathrm{NF}$ & A & $\mathrm{NF}$ & A & NF \\
\hline completa & 16 & 7 & 21,5 & 9 & 32 & 12 & 50 & 18 & 70 & 21 & 111 & 24 \\
\hline sem N & 15 & 8 & 19,5 & 10 & 23 & 12 & 24 & 13 & 27 & 15 & 44 & 17 \\
\hline sem $P$ & 17 & 8 & 23,5 & 10 & 36,5 & 14 & 42 & 14 & 64 & 17 & 76 & 17 \\
\hline sem K & 15 & 7 & 22,5 & 9 & 33 & 11 & 35 & 13 & 42 & 15 & 43 & 16 \\
\hline $\operatorname{sem} \mathrm{Ca}$ & 18 & 7 & 19,0 & 9 & 20 & 12 & 23 & 13 & 24 & 13 & 25 & 13 \\
\hline sem $\mathrm{Mg}$ & 16 & 8 & 22,0 & 9 & 23 & 13 & 24 & 14 & 24 & 15 & 25 & 17 \\
\hline sem S & 13 & 8 & 19,0 & 12 & 25 & 15 & 30 & 20 & 51 & 21 & 72 & 22 \\
\hline
\end{tabular}

$A=$ altura $; \mathrm{NF}=$ número de folhas

\section{Matéria seca}

Os resultados relacionados com a produção de matéria seca são apresentados no Quadro 3, e para a discussão dos mesmos separou-se raízes e parte aérea.

\section{Raizes}

Os tratamentos correspondentes as soluções completas, -S e -P apresentaram um sistema radicular com maior desenvolvimento quando comparado com os demais tratamentos. Por sua vez, o tratamento $-\mathrm{N}$, apresentou um sistema radicular muito reduzido, podendo isto ser explicado pelo efeito do nutriente no desenvolvimento da planta.

A análise da variância dos resultados obtidos revelou significância estatística en tre os tratamentos ao nível de $1 \%$ de probabilidade. A comparação das médias pelo teste de Tukey mostrou que o tratamento completo diferiu ao nivel de $1 \%$ de probabilidade dos demais tratamentos e que os tratamentos -S, -P e -Ca não diferiram entre si, mas apresentaram diferença significativa ao mesmo nível de probabilidade em relação aos tratamentos $-\mathrm{Mg},-\mathrm{K}$ e $-\mathrm{N}$. No Quadro 4 são encontrados os resultados da análise estatística.

De acordo com Bair, citado por NELSON (1956) a acumulação da matéria seca em função do tempo no milho tende a seguir as características de uma curva sigmóide. Entretanto os dados, do presente trabalho, não permitem uma comparação visto que a produção de matéria seca tanto das raízes como da parte aérea, só foram obtidas no final do experimento. 
QUADRO 3 - Produção de matéria seca por plantas de milho (Zea mays L.) cv. Piranão cultivadas em solução nutritiva.

\begin{tabular}{|c|c|c|c|c|c|c|c|}
\hline \multirow{3}{*}{ Tratamentos } & \multicolumn{6}{|c|}{ Parte Aérea } & \multirow[b]{3}{*}{ Kaiz } \\
\hline & \multicolumn{2}{|c|}{ Folhas } & \multirow[b]{2}{*}{ Colmc } & \multirow[b]{2}{*}{ Pendão } & \multirow[b]{2}{*}{ Espiga } & \multirow[b]{2}{*}{ Total } & \\
\hline & superiores & inferiores & & & & & \\
\hline \multirow{2}{*}{ completo } & 20,77 & 24,13 & 118,42 & 7,00 & 170,32 & 215,10 & 33,02 \\
\hline & 16,54 & 19,04 & 93,23 & 3,70 & 32,51 & 207,28 & 44,89 \\
\hline \multirow{2}{*}{$-\mathrm{N}$} & 1,95 & 5,75 & 3,38 & 0,18 & - & 11,26 & 5,84 \\
\hline & 3,21 & 7,25 & 8,63 & $\hat{1}, 14$ & -- & 20,23 & 7,34 \\
\hline \multirow{2}{*}{$-\mathbf{P}$} & 6,64 & 5,79 & 11,28 & 0,72 & - & 11,06 & 29,88 \\
\hline & 12,09 & 11,22 & 21,17 & 4,42 & - & 23,12 & 43,89 \\
\hline \multirow{2}{*}{$-\mathrm{K}$} & 10,70 & 11,70 & 12,50 & 1,80 & - & 36,77 & 7,70 \\
\hline & 11,73 & 8,73 & 10,53 & 5,81 & - & 36,80 & 5,89 \\
\hline \multirow{2}{*}{$-\mathrm{Ca}$} & 5,59 & 7,24 & 9,24 & - & - & 22,07 & 10,01 \\
\hline & 5,46 & 5,09 & 8,40 & - & - & 18,95 & 10,43 \\
\hline \multirow{2}{*}{$-\mathrm{Mg}$} & 8,97 & 13,17 & 8,16 & - & - & 30,30 & 9,87 \\
\hline & 16,07 & 9,58 & 7,49 & - & - & 33,14 & 8,39 \\
\hline \multirow{2}{*}{$-S$} & 10,38 & 19,60 & 126,86 & 4,72 & 159,97 & 214,68 & 29,98 \\
\hline & 18,94 & 18,32 & 72,35 & 3,13 & 114,33 & 170,93 & 24,86 \\
\hline
\end{tabular}

QUADRO 4 - Resultados da análise da variância e comparação das médias pelo teste Tukey na matéria seca das raízes (gramas).

\begin{tabular}{lrrr}
\hline & \multicolumn{2}{c}{ Repetições } & Médias \\
\cline { 2 - 4 } Tratamentos & \multicolumn{1}{c}{$\mathrm{I}$} & $\mathrm{II}$ & $38,95 \mathrm{a}$ \\
\hline \hline Completo & 44,89 & 33,02 & $6,59 \mathrm{c}$ \\
$-\mathrm{N}$ & 7,34 & 5,84 & $17,09 \mathrm{~b}$ \\
$-\mathrm{P}$ & 23,12 & 11,06 & $6,79 \mathrm{~b}$ \\
$-\mathrm{K}$ & 5,89 & 7,70 & $10,22 \mathrm{c}$ \\
$-\mathrm{Ca}$ & 10,43 & 10,01 & $8,88 \mathrm{c}$ \\
$-\mathrm{Mg}$ & 8,39 & 9,37 & $27,42 \mathrm{~b}$ \\
$-\mathrm{S}$ & 29,98 & 24,86 & \\
\hline $\mathrm{F}=13,36^{* *}$ & DMS $(1 \%)=20,58$ & $\mathrm{CV}=29 \%$ &
\end{tabular}

\section{Parte aérea}

Os dados de matéria seca referente à parte aérea encontram-se no Quadro 2, e os resultados da análise estatística dos mesmos aparecem no Quadro 5.

A análise de variância revelou uma diferença altamente significativa entre os tratamentos. A comparação das médias pelo teste de Tukey mostrou que os tratamentos completo e -S não diferem entre si; no entanto apresentam diferença significativa ao nivel de $1 \%$ de probabilidade quando comparados aos demais tratamentos, os quais são iguais entre si. 
QUADRO 5 - Resultados da análise da variância e comparação das médias pelo teste de Tukey na matéria seca da parte aérea (gramas).

\begin{tabular}{|c|c|c|c|}
\hline \multirow[b]{2}{*}{ Tratamentos } & \multicolumn{2}{|c|}{ Repetiçc̃es } & \multirow{2}{*}{ Médias } \\
\hline & I & II & \\
\hline Completo & 207,28 & 215,10 & $211,19 \mathrm{a}$ \\
\hline$-\mathrm{N}$ & 20,23 & 11,26 & $15,74 \mathrm{c}$ \\
\hline$-P$ & 43,89 & 29,88 & $36,88 \mathrm{c}$ \\
\hline$-\mathrm{K}$ & 36,80 & 36,07 & $36,43 \mathrm{c}$ \\
\hline$-\mathrm{Ca}$ & 18,95 & 22,07 & $20,51 \mathrm{c}$ \\
\hline$-\mathrm{Mg}$ & 33,14 & 30,30 & $31,72 \mathrm{c}$ \\
\hline$-\mathrm{S}$ & 170,93 & 214,68 & $192,80 \mathrm{a}$ \\
\hline $\mathrm{F}=76,92 * *$ & DMS (1 & $C V=16$ & \\
\hline
\end{tabular}

\section{Análise qu Imica}

Nitrogênio - Os resultados da determinação de $\mathrm{N}$ total nas folhas superiores e inferiores são apresentados no Quadro 6. A análise da variância mostrou efeito altamente significativo entre os tratamentos. No entanto, não foi observado significância estatística entre as folhas superiores e inferiores, o que era de se esperar, tendo em vista a facilidade de translocação do $\mathrm{N}$ na planta.

QUADRO 6 - Médias e resultados da análise da variância. Porcentagem de $\mathrm{N}$ nos tratamentos.

\begin{tabular}{lcc}
\hline \multirow{2}{*}{ Tratamentos } & \multicolumn{2}{c}{ Médias de 2 repetições } \\
\cline { 2 - 3 } & folhas inferiores & folhas superiores \\
\hline \hline Completo & 2,76 & 2,52 \\
$-\mathrm{N}$ & 1,84 & 1,22 \\
$-\mathrm{P}$ & 4,65 & 4,68 \\
$-\mathrm{K}$ & 4,64 & 3,78 \\
$-\mathrm{Ca}$ & 4,15 & 4,39 \\
$-\mathrm{Mg}$ & 5,15 & 4,79 \\
$-\mathrm{S}$ & 3,05 & 2,70 \\
\hline
\end{tabular}

$\mathrm{F}=7,04 * * \quad \operatorname{DMS}(1 \%)=2,01 \quad \mathrm{CV}=18 \%$

Convém salientar que as plantas dos tratamentos completo e -S continham menos nitrogênio do que as plantas dos dernais tratamentos, excetuando-se o tratamento $-\mathrm{N}$. 
Fósforo - Os resultados da determinação de $\mathrm{P}$ nas folhas superiores e inferiores são apresentados no Quadro 7.

QUADRO 7 - Médias e resultados da análise da variância. Porcentagem de P nos tratamentos.

\begin{tabular}{lcc}
\hline \multirow{2}{*}{ Tratamentos } & \multicolumn{2}{c}{ Médias de 2 repetições } \\
\cline { 2 - 3 } & folhas inferiores & folhas superiores \\
\hline \hline Completo & 0,59 & 0,53 \\
$-\mathrm{N}$ & 0,84 & 0,63 \\
$-\mathrm{P}$ & 0,14 & 0,17 \\
$-\mathrm{K}$ & 1,56 & 1,23 \\
$-\mathrm{Ca}$ & 1,32 & 1,09 \\
$-\mathrm{Mg}$ & 0,84 & 1,34 \\
$-\mathrm{S}$ & 0,59 & 0,56 \\
\hline
\end{tabular}

$\mathrm{F}=39,85 * * \quad \mathrm{DMS}(1 \%)=0,26 \quad \mathrm{CV}=12 \%$

A análise da variância revelou efeito significativo ao nível de $1 \%$ de probabilidade para os tratamentos, não havendo interação significativa entre as partes e tratamentos. Estes resultados revelam a translocação fácil do $\mathrm{P}$ na planta, concordando com o trabalho de BREWER \& BRAMLEY (1940) os quais detectaram P radioativo em folhas de milho, após 5 minutos de absorção.

Potássio - Os resultados da determinação de $\mathrm{K}$ nas folhas superiores e inferiores são apresentados no Quadro 8.

QUADRO 8 - Médias e resultados da análise da variância. Porcentagem de K nos tratamentos.

\begin{tabular}{lcc}
\hline & \multicolumn{2}{c}{ Médias de 2 repetições } \\
\cline { 2 - 3 } Tratamentos & folhas inferiores & folhas superiores \\
\hline \hline Completo & 2,25 & 2,26 \\
$-\mathrm{N}$ & 3,48 & 3,66 \\
$-\mathrm{P}$ & 4,45 & 3,31 \\
$-\mathrm{K}$ & 0,29 & 0,78 \\
$-\mathrm{Ca}$ & 4,45 & 4,17 \\
$-\mathrm{Mg}$ & 5,77 & 5,86 \\
$-\mathrm{S}$ & 3,29 & 2,61 \\
\hline
\end{tabular}

$F=29,62^{* *} \quad$ DMS $(1 \%)=2,19 \quad \mathrm{CV}=24 \%$

A análise de variância mostrou diferença significativa para os tratamentos ao nivel de $1 \%$ de probabilidade. A interação entre as partes e tratamentos não, foi significativa o que sugere a translocação do elemento ha planta, confirmando o que existe na literatura (NELSON, 1956). NOVAIS et al. (1971) trabalhando com híbridos de milho concluem que o AG 206 apesar do menor porte de suas plantas e da maior absorção de potássio apresentou acamamento. 
Cálcio - Os resultados da determinação do Ca nas folhas superiores e inferiores são apresentados no Quadro 9.

QUADRO 9 - Médias e resultados da análise da variância. Porcentagem de Ca nos tratamentos.

\begin{tabular}{lcc}
\hline & \multicolumn{2}{c}{ Médias de 2 repetições } \\
\cline { 2 - 3 } Tratamentos & folhas inferiores & folhas superiores \\
\hline Completo & 2,17 & 1,85 \\
$-\mathrm{N}$ & 0,48 & 0,22 \\
$-\mathrm{P}$ & 1,16 & 0,65 \\
$-\mathrm{K}$ & 2,04 & 1,16 \\
$-\mathrm{Ca}$ & 0,29 & 0,05 \\
$-\mathrm{Mg}$ & 0,80 & 0,59 \\
$-\mathrm{S}$ & 1,98 & 1,25 \\
\hline
\end{tabular}

$\mathrm{F}=29,62 * * \quad \operatorname{DMS}(1 \%)=0,53 \quad \mathrm{CV}=18 \%$

A análise da variância revelou significação estatística ao nível de $1 \%$ de probabilidade para os tratamentos. Embora a interação entre as partes e tratamentos não tenha revelado significação estatística, os dados encontrados sugerem a não translocação do Ca, concordando com MALAVOLTA (1970), entre outros.

Magnésio - Os resultados da determinação do $\mathrm{Mg}$ nas folhas superiores e inferiores são apresentados no Quadro 10.

QUADRO 10 - Médias e resultados da análise da variância. Porcentagem de Mg nos tratamentos.

\begin{tabular}{lcc}
\hline & \multicolumn{2}{c}{ Médias de 2 repetições } \\
\cline { 2 - 3 } Tratamentos & folhas inferiores & folhas superiores \\
\hline Completo & 0,82 & 0,66 \\
$-\mathrm{N}$ & 0,31 & 0,23 \\
$-\mathrm{P}$ & 0,62 & 0,37 \\
$-\mathrm{K}$ & 2,01 & 1,24 \\
$-\mathrm{Ca}$ & 1,15 & 0,85 \\
$-\mathrm{Mg}$ & 0,09 & 0,05 \\
$-\mathrm{S}$ & 0,94 & 0,80 \\
\hline $\mathrm{F}=15,56^{* *}$ & DMS $(1 \%)=0,51$ & $\mathrm{CV}=26 \%$
\end{tabular}

A análise da variância mostrou diferença significativa para os tratamentos, não havendo diferença significativa entre partes e tratamentos, o que demonstra sua translocação fácil. Estes dados concordam com os encontrados por Ciferri, citado por MiALAVOLTA (1970).

Enxofre - Os resultados da determinação do enxofre nas folhas superiores e inferiores são apresentados no Quadro 11 . 
QUADRO 11 - Médias e resultados da análise da variância. Porcentagem de S nos tratamentos.

\begin{tabular}{lcc}
\hline \multirow{2}{*}{ Tratamentos } & \multicolumn{2}{c}{ Médias de 2 repetições } \\
\cline { 2 - 3 } & folhas inferiores & folhas superiores \\
\hline \hline Completo & 0,38 & 0,32 \\
$-\mathrm{N}$ & 0,35 & 0,25 \\
$-\mathrm{P}$ & 0,21 & 0,21 \\
$-\mathrm{K}$ & 0,17 & 0,15 \\
$-\mathrm{Ca}$ & 0,22 & 0,27 \\
$-\mathrm{Mg}$ & 0,13 & 0,11 \\
$-\mathrm{S}$ & 0,31 & \\
\hline $\mathrm{F}=8,25^{* *}$ & $\mathrm{CV}=17 \%$ & 0,26 \\
\hline
\end{tabular}

A análise da variância mostrou diferença significativa ao nível de $1 \%$ de probabilidade para os tratamentos. Entretanto, não houve diferença estatística entre partes e tratamentos. Isto pode ser atribuído ao fato das plantas terem sido pulverizadas com Thiordan (inseticida à base de S).

\section{Comparação dos teores dos macronutrientes em cada tratamento estudado}

Os resultados relacionados com folhas superiores e inferiores são encontrados nos Quadros 12 a 18.

QUADRO 12 - Médias e resultados da análise da variância. Macronutrientes no tratamento completo.

\begin{tabular}{lcc}
\hline \multirow{2}{*}{ Tratamentos } & \multicolumn{2}{c}{ Médias de 2 repetições } \\
\cline { 2 - 3 } & folhas inferiores & folhas superiores \\
\hline $\mathrm{N}$ & 2,67 & 2,52 \\
$\mathrm{P}$ & 0,69 & 0,53 \\
$\mathrm{~K}$ & 2,25 & 2,26 \\
$\mathrm{Ca}$ & 2,17 & 1,85 \\
$\mathrm{Mg}$ & 0,82 & 0,66 \\
$\mathrm{~S}$ & 0,38 & 0,32 \\
\hline
\end{tabular}

$\mathrm{F}=102,23^{* *} \quad \operatorname{DMS}(1 \%)=0,35 \quad \mathrm{CV}=9 \%$


QUADRO 13 - Médias e resultados da análise da variância. Macronutrientes no tratamento $-\mathrm{N}$.

\begin{tabular}{lcc}
\hline \multirow{2}{*}{ Tratamentos } & \multicolumn{2}{c}{ Médias de 2 repetições } \\
\cline { 2 - 3 } & folhas inferiores & folhas superiores \\
$\mathbf{P}$ & 1,84 & 1,22 \\
$\mathrm{~K}$ & 0,84 & 0,63 \\
$\mathrm{Ca}$ & 3,48 & 3,66 \\
$\mathrm{Mg}$ & 0,48 & 0,2 \\
$\mathrm{~S}$ & 0,31 & 0,2 \\
\hline $\mathrm{F}=27,84^{* *}$ & 0,35 & 0 \\
\end{tabular}

QUADRO 14 - Médias e resultados da análise da variância. Macronutrientes no tratamento $-P$.

\begin{tabular}{lcc}
\hline \multirow{2}{*}{ Tratamentos } & \multicolumn{2}{c}{ Médias de 2 repetições } \\
\cline { 2 - 3 } & folhas inferiores & folhas superiores \\
$\mathrm{N}$ & 4,65 & 4,68 \\
$\mathrm{P}$ & 0,14 & 0,17 \\
$\mathrm{~K}$ & 4,45 & 3,31 \\
$\mathrm{Ca}$ & 1,16 & 0,65 \\
$\mathrm{Mg}$ & 0,62 & 0,37 \\
$\mathrm{~S}$ & 0,21 & 0,21 \\
\hline $\mathrm{F}=5,03 * *$ & $\mathrm{DMS}(1 \%)=3,35$ & $\mathrm{CV}=71 \%$
\end{tabular}

QUADRO 15 - Médias e resultados da análise da variância. Macronutrientes no tratamento $-\mathrm{K}$.

\begin{tabular}{lcc}
\hline \multirow{2}{*}{ Tratamentos } & \multicolumn{2}{c}{ Médias de 2 repetições } \\
\hline $\mathrm{N}$ & folhas inferiores & folhas superiores \\
$\mathrm{P}$ & 4,64 & 3,78 \\
$\mathrm{~K}$ & 1,56 & 1,23 \\
$\mathrm{Ca}$ & 0,29 & 0,78 \\
$\mathrm{Mg}$ & 2,04 & 1,16 \\
$\mathrm{~S}$ & 2,01 & 1,24 \\
\end{tabular}

$\mathbf{F}=20,08 * *$

$\operatorname{DMS}(1 \%)=1.20$

$\mathrm{CV}=28 \%$ 
1 QUADRO 16 - Médias e resultados da análise da variância. Macronutrientes no tratamento $-\mathrm{Ca}$.

\begin{tabular}{lcc}
\hline \multirow{2}{*}{ Tratamentos } & \multicolumn{2}{c}{ Médias de 2 repetições } \\
\cline { 2 - 3 } & folhas inferiores & folhas superiores \\
\hline $\mathbf{N}$ & 4,15 & 4,39 \\
$\mathbf{P}$ & 1,32 & 1,09 \\
$\mathrm{~K}$ & 4,45 & 4,17 \\
$\mathrm{Ca}$ & 0,29 & 0,05 \\
$\mathrm{Mg}$ & 1,15 & 1,35 \\
$\mathrm{~S}$ & 0,22 & 0,27 \\
\hline
\end{tabular}

$F=23,18^{* *} \quad \operatorname{DMS}(1 \%)=1,46 \quad C V=29 \%$

QUADRO 17 - Médias e resultados da análise da variância. Macronutrientes no tratamento - Mg.

\begin{tabular}{lcc}
\hline \multirow{2}{*}{ Tratamentos } & \multicolumn{2}{c}{ Médias de 2 repetições } \\
\cline { 2 - 3 } & folhas inferiores & folhas superiores \\
\hline $\mathbf{N}$ & 5,15 & 4,79 \\
$\mathrm{P}$ & 0,84 & 1,34 \\
$\mathrm{~K}$ & 5,77 & 5,86 \\
$\mathrm{Ca}$ & 0,80 & 0,59 \\
$\mathrm{Mg}$ & 0,09 & 0,05 \\
$\mathrm{~S}$ & 0,13 & 0,11 \\
\hline $\mathrm{F}=102,06^{* *}$ & $\mathrm{DMS}(1 \%)=0,94$ & $\mathrm{CV}=16 \%$
\end{tabular}

QUADRO 18 - Médias e resultados da análise da variância. Macronutrientes no tratamento $\mathrm{S}$.

\begin{tabular}{lcc}
\hline \multirow{2}{*}{ Tratamentos } & \multicolumn{2}{c}{ Médias de 2 repetições } \\
\cline { 2 - 3 } $\mathbf{N}$ & folhas inferiores & folhas superiores \\
$\mathbf{P}$ & 3,05 & 2,70 \\
$\mathbf{K}$ & 0,59 & 0,56 \\
$\mathbf{C a}$ & 3,29 & 2,61 \\
$\mathbf{M g}$ & 1,98 & 1,75 \\
$\mathbf{S}$ & 0,94 & 0,80 \\
$\mathbf{F}=18,70^{* *}$ & 0,31 & 0,26 \\
\hline
\end{tabular}


Pode-se observar que, quando se omitiu $\mathrm{N}$, houve diminuição nos teores de $\mathrm{Ca}$ e $\mathrm{Mg}$ e aumento no teor de K.

Resultados semelhantes foram encontrados por BECKENBACH et al. (1938) os quais mostraram que os teores de $\mathrm{N}$ no substrato. Wadleigh \& Shive, citados por NEL. SON (1956), notaram que o íon amônio diminui a absorção de $\mathrm{K}$, resultados que estão de acordo com os encontrados no presente trabalho.

Por sua vez, quando o $\mathrm{P}$ foi omitido o teor de $\mathrm{N}$ nas folhas quase se duplicou.

Embora BECKENBACH et al. (1938) tenham concluído que a absorção de P não é efetuada pela variação na concentração de outros ions no substrato, foi observado neste trabalho que quando se omitiu $\mathrm{K}$ o teor de $\mathrm{P}$ subiu.

AGBIN (1968) mostrou que a presença de $\mathrm{P}$ aumentava a absorção de Ca e $\mathrm{Mg}$, resultados coerentes com os encontrados neste experimento.

UDOVENKO \& BEZLYUDNYI (1971) demonstraram que o conteúdo de $\mathrm{P}$ na planta depende da forma em que $\mathrm{N}$ foi fornecido, e que quando este era aplicado na forma de nitrato ocorria inibição na absorção de $\mathrm{P}$.

Por outro lado, quando se omitiu K ocorreu um aumento considerável no teor de $\mathrm{Mg}$ das folhas.

Os teores de $\mathrm{K}$ e de $\mathrm{Mg}$ nas folhas aumentaram consideravelmente quando se omitiu $\mathrm{Ca}$. Entretanto, quando a omissão foi de $\mathrm{Mg}$, houve aumento de $\mathrm{K}$ nas folhas.

THOMAS \& MACK (1939) encontrou que a elevada absorção de K diminuía a absorção de $\mathrm{Ca}$ e $\mathrm{Mg}$, dados estes confirmados no presente trabalho.

\section{Descrição dos sintomas de deficiências}

Nitrogênio - Planta pouco desenvolvida apresentando clorose generalizada a qual é iniciada nas folhas mais velhas, progredindo para as mais novas. À medida que o tecido amarelado seca, toma o aspecto de queimado. Note-se nas folhas, particularmente nas mais velhas um "V" amarelado cujo vértice coincide com a nervura principal na base da folha.

Fósforo - Desenvolvimento quase idêntico ao completo, mostrando as folhas coloração inicialmente verde-escuro, tornando-se, posteriormente, roxas nas pontas e margens. $\mathrm{O}$ colmo também apresentou coloração roxa.

Potássio - Plantas pouco desenvolvidas com - folhas apresentando necrose nas margens e pontas (inicialmente nas mais velhas e posteriormente nas mais novas). Neste tratamento apareceram sintomas característicos da deficiência de Fe (clorose internerval).

Cálcio - Plantas pouco desenvolvidas, apresentando sintomas iniciais na parte superior, caracterizados por: amarelecimento, secamento, necrose e dilaceração das margens, clorose internerval (faixas largas) e morte da região de crescimento. 
Magnésio - Os sintomas se caracterizam por atrofiamento das plantas, apresentando as folhas inferiores clorose internerval progredindo posteriormente para as folhas superiores.

Enxofre - As plantas apresentaram crescimento próximo ao normal, não exibindo suas folhas sintomas aparentes de deficiência.

Segundo NELSON (1956) os sintomas de deficiência de enxofre em milho, não tem sido descritos na literatura, embora sejam freqüentes as respostas à aplicação deste elemento.

\begin{tabular}{|c|c|c|c|c|c|c|c|c|c|c|c|c|}
\hline \multirow{2}{*}{ Tratamentos } & \multicolumn{2}{|c|}{ N\% } & \multicolumn{2}{|c|}{$\mathrm{p} \%$} & \multicolumn{2}{|c|}{$K \%$} & \multicolumn{2}{|c|}{$\mathrm{Ca} \%$} & \multicolumn{2}{|c|}{$\mathrm{Mg} \%$} & \multicolumn{2}{|c|}{$-\quad \mathrm{S} \%$} \\
\hline & int. & sup. & inf. & sup. & in! & sup. & inf & sup. & int. & sup. & $\ln t$ & sup. \\
\hline \multirow{2}{*}{ Completo } & 2.52 & 2,41 & 0,66 & 0.53 & 2.33 & 2.49 & 2,13 & 1,82 & 0.85 & 0,68 & 0.38 & 0. 32 \\
\hline & 2,83 & 2,63 & 0.53 & 0.53 & 2,17 & 2.03 & 2.21 & 1,88 & 0.80 & 0.65 & 0.38 & 0,32 \\
\hline \multirow{2}{*}{1} & 1.59 & 1.24 & 0.83 & 0,74 & 3,88 & 4,33 & 0.43 & 0,30 & 0,31 & 0.25 & 0.33 & 0.22 \\
\hline & 2.09 & 1,20 & 0,86 & 0.53 & 3.08 & 3.00 & 0,54 & 0,15 & 0.32 & 0.22 & 0.38 & 0.28 \\
\hline \multirow[t]{2}{*}{$P$} & 5.53 & 5.03 & 0.13 & 0.18 & 5.76 & 4.35 & 1.04 & 0,59 & 0,72 & 0.40 & 0.27 & 0.15 \\
\hline & 3,77 & 4.33 & 0.15 & 0.16 & 3.14 & 2.28 & 1.29 & 0,71 & 0.52 & 0,34 & 0.27 & 0.16 \\
\hline \multirow[t]{2}{*}{ K } & 5.09 & 3.03 & 1.58 & 1.40 & $(.35$ & 1.14 & 2.19 & 1.49 & 2.20 & 1.49 & 0.16 & 0.11 \\
\hline & 4.19 & 4.53 & 1.54 & 1.06 & 0.23 & 0,42 & 1,90 & 0.83 & 1,82 & 1.00 & 0.19 & 0.22 \\
\hline \multirow[t]{2}{*}{$\mathrm{Ca}$} & 4,14 & 3.55 & 1.43 & 1.03 & 4.08 & 4.39 & 0,33 & 0.06 & 0,12 & 0.74 & 0.22 & 0,27 \\
\hline & 4.17 & 5.23 & , 1,22 & 1.16 & 4.82 & 3.95 & 0.26 & 0.04 & 1,18 & 0.97 & 0.22 & 0.27 \\
\hline \multirow[t]{2}{*}{$-\mathbf{M g}$} & 5.82 & 4.51 & 0.88 & 1.36 & 6.13 & 6.08 & 0.89 & 0.63 & 0,10 & 0.06 & 0.11 & 0,11 \\
\hline & 4,49 & 5,08 & 0.80 & 1.32 & 5.42 & 5.64 & 0.71 & 0.55 & 0.08 & 0.05 & 0.16 & 0.11 \\
\hline \multirow{2}{*}{$S$} & 3,01 & 2.44 & 0.66 & 0,60 & 4,01 & 2.57 & 2.04 & 2,05 & 1,13 & 1.10 & 0.32 & 0.27 \\
\hline & 3.10 & 2.96 & 0.53 & 0.53 & 2.57 & 2.65 & 1.93 & 1,45 & 0.75 & 0,50 & 0.31 & 0.26 \\
\hline
\end{tabular}

QUADRO 19 - Resultados das análises químicas em folhas inferiores e superiores de plantas de milho (Zea mays L.) cv. Piranão.

\section{RESUMO E CONCLUSÕES}

1 - Com a finalidade de obter um quadro sintomatológico das deficiências de macronutrientes no milho, variedade Piranão, as plantas foram cultivadas em solução nutritiva completa e com omissão de um elemento por vez.

2 - Os sintomas obtidos foram os seguintes:

2.1. Nitrogênio - plantas pouco desenvolvidas; folhas com clorose em "V" que se inicia nas mais velhas, seguida de secamento e necrose;

2.2. Fósforo - coloração verde-escura das folhas mais velhas com tonalidades roxas.

2.3. Potássio - plantas pouco desenvolvidas; folhas mais velhas com clorose e necrose das pontas e margens; folhas mais novas com clorose devida à falta de ferro.

2.4. Cálcio - plantas muito pouco desenvolvidas; morte da região de crescimento; folhas mais novas com clorose, necrose e dilaceração das margens e pontas e lâmina com largas faixas longitudinais esbranquiçadas.

2.5. Magnésio - plantas atrofiadas; folhas velhas com clorose intemerval, sintoma que progride para as folhas superiores.

2.6. Enxofre - ausência de sintomas marcantes. 
3 - A produção de matéria seca obedecem à seguinte ordem decrescente:

$$
\text { completo }>-\mathrm{S}>\cdot \mathrm{P}=-\mathrm{K}>-\mathrm{Mg}>-\mathrm{N}>-\mathrm{Ca} ;
$$

as plantas $-\mathrm{N},-\mathrm{P},-\mathrm{K},-\mathrm{Ca}$ e $-\mathrm{Mg}$ não deram espigas; relação parte aérea/raiz decresceu na seguinte ordem:

$$
-\mathrm{S}>\text { completo }=-\mathrm{K}>-\mathrm{Mg}>-\mathrm{N}>-\mathrm{P}=-\mathrm{Ca}
$$

4 - Os teores dos elementos encontrados no tratamento completo foram os seguintes, respectivamente para folhas inferiores e superiores: $\mathrm{N}-2,76$ e $2,52 \% ; \mathrm{P}-0,59$ e $0,53 \% ; \mathrm{K}-2,25$ e $2,26 \%$; $\mathrm{Ca}-2,17$ e $1,85 \% ; \mathrm{Mg}-0,82$ e $0,66 \% ; \mathrm{S}-0,38$ e $0,32 \%$; os teores encontrados nas plantas com a deficiência correspondente foram, respectivamente para as folhas inferiores e superiores: $\mathrm{N}-1,84$ e $1,22 \% ; \mathrm{P}-0,14$ e $0,17 \% ; \mathrm{K}-0,29$ e $0,78 \%$; $\mathrm{Ca}-0,29$ e $0,05 \% ; \mathrm{Mg}-0,09$ e $0,05 \% ; \mathrm{S}-0,31$ e $0,26 \%$.

\section{SUMMARY}

\section{STUDIES ON THE MINERAL NUTRITION OF CORN. II. DEFICIENCIES OF MACRONUTRIENTS IN THE VARIETY PIRANÃO}

Corn plants, Piranão variety, were grown in nutrient solution, complete and with omission of one macronutrient each time, in order to gain information on the effects of treatments on growth, symptoms of deficiency, and mineral composition of the leaves.

The following symptoms of deficiency were obtained:

Nitrogen -- plants underdeveloped; "V" shaped chlorosis beginning in the older leaves followed by drying and necrosis;

Phosphorus - older leaves dark green with a purple hve;

Potassium - plants underdeveloped; marginal chlorisis and necrosis in the older leaves; iron induced chlorosis in the younger leaves;

Calcium - plants very underdeveloped due to death of apical merystem; younger leaves showing tip and marginal chlorosis and afterwards necrosis and break down of the tissue; wide whitish areas in the younger leaves;

Magnesium - plants very underdeveloped; internerval chlorosis in older leaves first, proceeding to the young ones; leaves.

Sulfur - absence of marked symptoms, except for a slight chlorosis in the younger 
Dry matter production obeyed the following decreasing order:

Complete, minus $\mathrm{S}$, minus $\mathrm{P}$, minus $\mathrm{K}$, minus $\mathrm{N} / \mathrm{g}$, minus $\mathrm{N}$ and minus $\mathrm{Ca}$.

Ears were obtained only in treatments complete and minus $\mathrm{S}$.

Top/root ratio decreased according to this order:

minus $\mathrm{S}$, complete, minus $\mathrm{K}$, minus $\mathrm{Mg}$, minus $\mathrm{N}$, minus $\mathrm{P}$ and minus $\mathrm{Ca}$.

The macronutrient content for the treatment complete was the following respectively for lower and upper, leaves: $\mathrm{N}-2.76$ and $2.52 \% ; \mathrm{P}-0.59$ and $0.53 \% ; \mathrm{K}-2.25$ and $2.26 \% ; \mathrm{Ca}-2.17$ and $1.85 ; \mathrm{Mg}-0.82$ and $0.66 ; \mathrm{S}-0.38$ and 0.32 . The values of the element corresponding to each treatment were: $\mathrm{N}-1.84$ and 1.22 (that is: lower leaves in the minus $\mathrm{N}$ plants had $184 \% \mathrm{~N}$, whereas the upper leaves in same treatment had $1.22 \% \mathrm{~N} ; \mathrm{P}-0.14$ and $0.17 \% ; \mathrm{K}-0.29$ and $0.78 \% ; \mathrm{Ca}-0.29$ and $0.05 ; \mathrm{Mg}-$ 0.09 and $0.05 \% ; \mathrm{S}-0.31$ and $0.26 \%$.

\section{LITERATURA CITADA}

AGBIM, N.N., 1968. Effect of Phosphorus and Magnesium on zin Nutrition of Corn. Thesis, Colorado State Univ., Fort Collins, $81 \mathrm{p}$.

AGBoOlA, A., 1967. The infiuence of Fertilizer Placement on the Yield of Maize. Nigeriam Agr. J., 4(1):32-34.

ANDERSON, G.D., 1970. Fertility Studies on a Sandy Loam in Semiarid Tanzania. I. Effects of Nitrogen, Phosphorus, and Potassium Fetilizers on Yields of Maize and Soil Nutrient Status. Exp. Agr., 6(1):1-12.

ANDRADE, A.G., 1975. Acumulação diferencial de nutrientes por cinco cultivares de milho (Zea mays L.). Diss. de Mestrado, E.S.A. "Luiz de Queiroz", USP, Piracicaba.

ARRUDA, H.V., 1968. Adubação mineral do milho nas terras roxas do município de Ribeirão Preto. O Biológico, 34(5) 99-100.

BECKENBACH, J.R., ROBINS, W.R. \& SHIVE, J.W., 1938. Nutrition Studies with corn. II. A Statistical Interpretation of the relation Between the ionic concentration of the culture solutions and the element content of the Tissues. Soil Sci., 45:403-426.

BRAGA, J.M., 1968. Avaliação da fertilidade de solos de Viçosa, Minas Gerais. Uso de Microparcelas. Rev. Ceres, 15(86):219-243.

BREWER, A.K. \& BRAMLEY, A., 1940. A radioactive isotope study of the absorption of phosphorus and sodium by corn seedlings. Science, $91: 269-270$.

GALLO, J.R. \& HIROCE, R., 1968. A análise foliar na nutrição do milho. I. Correlação entre análises de folhas e produção. Bragantia, 27(15):177-186.

GALVÃO, J.D., BRANDÃO, S.A. \& GOMES, F.R., 1969. Efeito da população de plantas e níveis de nitrogênio sobre a produção de grãos e sobre o peso médio das espigas. Experientiae, $9(2)$ : 39-82.

HOAGLAND, D.R. \& ARNON, D.I., 1950. The water culture method for growing plants without soil. Calif. Agr. Exp. Sta. Circ. 347.

LAIRS, R.J. \& RODRIGUEZ, G.J.H., 1965. Application of Fertilizers to wet-Season Maize in Areas of Guanajato. Michoacan and Jalisco States. Foll. Tecn. Inst. Nac. Invext. Agricolas, México, 50 :1-71. 
MALAVOLTA, E., 1970. Nutrição mineral de Plantas. ESALQ, Piracicaba, mim. 218 p.

MALIK, M.Y. \& SHAH, W.H., 1968. Effect of Nitrogen and Phosphorus Fertilizers on the Nutrient Contents and Yield of Maize. Park. J. Sci., 20(4):178-181.

MIRANDA, L.E.C. de \& MIRANDA, L.T., 1971. Adubação do milho. IV Estudo Econômico de Adubação do milho no Estado de São Paulo. Projeto BNDE/ANDA/CIA. Boletim no 14.

NAKANO, H. \& KUSHIBIKI, H., 1967. Studies on Magnesium Deficiency Symptom in Corn Crop. 1. Varietal Difference of Magnesium Deficiency Symptom and Effects of Magnesium Fertilizer on Growth and Yield in Corn. Bull. Hokkaido Prefect. Agr. Exp. Sta., 15:15-21.

NELSON, B.L., 1956. The mineral nutrition of corn as related to its growth and culture. Advances in Agronomy, 8:321-375.

NOVAIS, R.F., BRAGA, J.M., GALVÃO, D.J. \& GOMES, F.R., 1971. Efeito de nitrogênio em populações de plantas e híbridos sobre a produção de grãos e sobre algumas características agronômicas da cultura do milho., Experientiae, 12(10):341-381.

PUNTAMKER, S.S., MEHTA, K.M. \& SHARMA, V.C., 1965. Uptake of N, P and K by Maize in Relation to Levels of Nitrogen. Indian J. Agron., 10:244-252.

RATHORE, S.S. \& SINGH, H.G., 1967. Response of Maize to Increasing Levels of N and P at Varying Plant Densities. Univers. Udaipur res. Stud., $3: 29-33$.

REHM, G.W. \& CALDWELL, A.C., 1970. Effect of the Separation of Nitrogen and sulfur Sources on Sulfur Uptake by Corn. Proc. Soil Sci. Soc. Amer., 34(5):769-772.

SAXENA, M.C. \& GAUTAM, O.P., 1971. Response of Hybrid Maize to Levels of Nitrogenous and Phosphatic Fertilization. Nutrition of Maize. Bull. Y-26. Tenn. Valley Anthority.

SHAG, V.H., 1969. Fertilizer Needs of Maize. Indian Farming, 18(12):41-45.

SOBRAL, L.F., 1972. Efeito de doses crescentes de fósforo na produção do milho. IPEAL. Aracaju (não publicado).

THOMAS, W., \& MACK, W.B., 1939. The foliar diagnosis of Zea mays L., subjectid to differential fertilizer treatment. J. Agr. Research., 58:477-491.

UDOVENKO, G.V. \& BEZLYUDNYI, N.N., 1971. Effect of Nitrogen nutrition on growth and protein metabolism of plants. Em: Nutrition of Maize. Bull. Y-26, Tenn. Valley Anthority.

WALLACE, A. \& DEUTSCH, A., 1968. Phosphorus Deficiency Decreases Stomatal Activity and Water Use of Plants. Calif. Agr., 22(8):15.

T.V.A., 1971. Nutrition of Maize. Muscle Shoals. 
